\title{
Emerging intelligent algorithms: challenges and applications
}

\author{
Gunasekaran Manogaran ${ }^{1} \cdot$ Naveen Chilamkurti ${ }^{2}$ Ching-Hsien $\mathrm{Hsu}^{3}$
}

Published online: 18 December 2018

(C) Springer-Verlag London Ltd., part of Springer Nature 2018

The emerging edge computing technologies, IoT and rich cloud services are used to create a novel technology called Edge of Things (EoT). In EoT, data processing occurs in part at the network edge or between the cloud-to-end that can best meet customer necessities, rather than entirely processing in a comparatively less number of massive clouds. The main challenge in EoT is how to manage with emerging IoT environments, where a large number of connected devices participate for restricted wireless resources and where heterogeneity is ever-increasing. In order to overcome this issue, there is an urgent need for more intelligent algorithms and architectures that lead to more interoperable solutions and that can make effective decisions in emerging EoT. This special issue focuses on emerging intelligent algorithms' challenges and applications for IoT and EoT platforms. The selected papers are summarized as follows: Hassan et al. [1] have proposed HAAL-NBFA framework with a five-phase classification technique to handle big imbalanced datasets, resulting from long-term monitoring of elderly patients. In this paper, the firefly algorithm (FA) has been used to optimize naïve Bayes classifier (NB) which selects the minimum features that give the highest accuracy.

Batalla and Gonciarz [2] have proposed an architecture for smart home management system, implemented the necessary modules and tested it from the point of view of security and availability. The management is controlled by the network operator in a similar way as occurs with current set-top boxes for multimedia streaming at home. Liang

\footnotetext{
Gunasekaran Manogaran

gmanogaran@ucdavis.edu

Naveen Chilamkurti

n.chilamkurti@latrobe.edu.au

Ching-Hsien Hsu

chh@chu.edu.tw

University of California, Davis, Davis, USA

2 LaTrobe University, Melbourne, Australia

3 National Chung Cheng University, Chiayi, Taiwan
}

et al. [3] have proposed the gray clustering model which is the combination of improved gray clustering algorithm and principle of mechanical specific energy. They have introduced correlation degree analysis method; this model optimizes the original gray fixed weight clustering monitoring model, establishing a horizontal well-oriented grayrelated clustering model.

Amoon et al. [4] have proposed an algorithm to schedule applications' tasks to virtual machines (VMs) of cloud computing systems. The algorithm has three phases: level sorting, task prioritizing and virtual machine selection. The three-phase process successfully assigns the virtual machine for each task without making any difficulties for evaluating the algorithm performance; extensive simulation experiments are performed. Popa et al. [5] have proposed a modular platform that uses the power of cloud services to collect, aggregate and store all the data gathered from the smart environment.

Selvaraj and Sivaraman [6] have elucidated a data-predicting model using an intelligent rule-based enhanced multiclass support vector machine and fuzzy rules (IREMSVM-FR) while optimizing the test practices and trials needed for the proportioning of self-compacting concrete (SCC) using response surface methodology (RSM). Ma et al. [7] have proposed an improved dynamic self-adaption cuckoo search algorithm based on collaboration between subpopulations to improve convergence rate and optimization precision of the cuckoo search (CS) algorithm.

AlFarraj et al. [8] have introduced a method of optimized feature selection and soft computing techniques for reducing the dimensionality of the dataset. The optimized features were selected using the fireflies gravitational ant colony optimization (FGACO) approach. Prabhakaran and Sudhakar [9] have introduced a method of optimized feature selection and soft computing techniques for a midvehicle collision detection and avoidance system with a constraint-free condition that produces mid-vehicle maneuvers, particularly when jammed between the front and rear vehicles. 
Nagarajan and Gandhi [10] have proposed the hybrid sentiment analysis called ternary classification based on preprocessing technique, and the results of tweets sent by the users are obtained. Rizk-Allah et al. have proposed a new binary version of the SSA named BSSA based on a modified arctan transformation. This modification has two features regarding the transfer function, namely multiplicity and mobility [11]. Sun and Lu have proposed genetic algorithm (GA) method to qualify the population diversity and similarity between adjacent generations. The convergence speed and the global optimal solution are greatly improved [12].

Pan et al. have proposed a new and efficient firefly algorithm (namely NEFA). In NEFA, three modified strategies are employed. First, a new attraction model is used to determine the number of attracted fireflies. Second, a new search operator is designed for some better fireflies. Third, the step factor is dynamically updated during the iterations [13]. Jing and Zhang have proposed a immune clone selection algorithm for Optimization Model of Car Flow Organization. Besides, premature convergence can be prevented by using the antibody concentration which can control the population size [14].

Jancy and Jayakumar have proposed a framework for path construction phase (PCP) and alternative path construction phase (APCP) are created in order to reduce dead nodes. The proposed techniques are compared with EAMMH protocol and LEACH protocol using MATLAB [15]. Gu et al. have proposed Markov clustering algorithm based on link similarity (MLS). First of all, the weighted link similarity is calculated and the link similarity matrix which measures the association strength of the protein interactions can be gotten [16].

Ai et al. [17] have employed a deep learning approach, named the convolutional long short-term memory network (conv-LSTM), to address the spatial dependences and temporal dependences. Arivudainambi et al. [18] have proposed that detection technique is robust enough to detect DDoS attack within the least magnitude of attack traffic. Further, to evaluate the performance, the proposed method is compared with the state-of-the-art techniques.

Agasthian et al. [19] have proposed a method to decide the parameters for support vector machine (SVM) in wind turbine called cuckoo search optimization (CSO). The combination of optimization technique with classification technique is evaluated. MATLAB platform was used to evaluate the various faults under fixed value and gain factor conditions. Sasikala and Shoba Bindu [20] have solved the certificate management issue in PKI-based protocols and also provided security against quantum computer attacks; in this work, we design a Certificateless RDIC protocol using lattices. In this approach, the data integrity checking can be initiated using data owner's identity (his name or email address) along with some secret information, which can guarantee the right public key is used for RDIC.

Lokesh et al. [21] have proposed An Automatic Tamil Speech Recognition system by using Bidirectional Recurrent Neural Network with Self-Organizing Map-based classification scheme is suggested for Tamil speech recognition. Tamil digits and words are ordered by utilizing BRNN classifier where the settled length feature vector from SOM is given as input, named as BRNN-SOM. R. Dinesh Jackson Samuel and Rajesh Kanna [22] have proposed tuberculosis detection system consists of two subsystems-a data acquisition system and a recognition system. In the data acquisition system, a motorized microscopic stage is designed and developed to automate the acquisition of all FOVs. Here the microscopic stage movement is motorized and scanning patterns are defined by the user for specimen examination.

Al-Bashir et al. [23] have proposed an algorithm to measure the Cobb angle semiautomatically. The algorithm is based on two processing phases in which each column in the raw X-ray image is reduced to two points representing the end points of the spine and containing its general structure and outline. These points are then used to fit a fifth-order polynomial. Ding et al. [24] have proposed a systematic performance diagnosing method focusing on building an accurate and interpretable performance model with performance counters. Our method is able to diagnose the HPC application scaling issues by predicting its runtime and performance behaviors in different functions.

Altameem and Amoon [25] have proposed a novel big data and soft computing techniques for recognizing the crime activities with effective manner. The prediction process is done by using the enhanced associative neural networks approach.

Xing et al. [26] have built the BP neural network inverse model with multiple inputs and single output based on internal model control. Therefore, it realizes the inverse mapping between the output and the input variables of the BP neural network. Chandra Babu and Shantharajah [27] have proposed work is concerned in predicting the probability of CVD and high blood pressure in India. The disease has been predicted with body mass index value; from the health reports of India, the pervasiveness of CVD and HBP is identified.

Abdel-Basset et al. [28] have introduced the neutrosophic LP models where their parameters are represented with a trapezoidal neutrosophic numbers and presented a technique for solving them. Wang et al. [29] have proposed the sparse topic model is proposed to explore the latent motion patterns and achieve a sparse representation for the video scene and the semi-supervised learning method is applied to enhance the discrimination of model and improve the performance of anomaly detection. 
$\mathrm{Wu}$ and Zhang [30] have proposed a generalized additive model (GAM) is used in this paper to analyze the impact that different influence factors, especially their interaction, have on $\mathrm{PM}_{2.5}$ concentration and its diffusion process. Frank Vijay [31] has proposed hybrid technique which incorporates both quality factors and fuzzy-based technique in function point analysis, and it also evaluates the accuracy of fuzzy analysis for software effort estimation.

We would like to convey our sincere thanks to all the researchers for submitting their manuscripts and a special note of thanks to the reviewers, whose efforts have allowed the selection of good-quality papers. We are also grateful to the Neural Computing and Applications, for allowing us to divulge a selected sample of the ongoing research efforts on recent advancements in machine learning algorithms.

\section{References}

1. Hassan MK, El Desouky AI, Badawy MM et al (2018) EoTdriven hybrid ambient assisted living framework with naïve Bayes-firefly algorithm. Neural Comput Appl. https://doi.org/10. 1007/s00521-018-3533-y

2. Batalla JM, Gonciarz F (2018) Deployment of smart home management system at the edge: mechanisms and protocols. Neural Comput Appl. https://doi.org/10.1007/s00521-018-3545-7

3. Liang H, Sun Y, Li G et al (2018) Gray relational clustering model for intelligent guided monitoring horizontal wells. Neural Comput Appl. https://doi.org/10.1007/s00521-018-3645-4

4. Amoon M, El-Bahnasawy N, ElKazaz M (2018) An efficient cost-based algorithm for scheduling workflow tasks in cloud computing systems. Neural Comput Appl. https://doi.org/10. 1007/s00521-018-3610-2

5. Popa D, Pop F, Serbanescu C et al (2018) Deep learning model for home automation and energy reduction in a smart home environment platform. Neural Comput Appl. https://doi.org/10. 1007/s00521-018-3724-6

6. Selvaraj S, Sivaraman S (2018) Prediction model for optimized self-compacting concrete with fly ash using response surface method based on fuzzy classification. Neural Comput Appl. https://doi.org/10.1007/s00521-018-3575-1

7. Ma H, Li S, Li S et al (2018) An improved dynamic self-adaption cuckoo search algorithm based on collaboration between subpopulations. Neural Comput Appl. https://doi.org/10.1007/ s00521-018-3512-3

8. AlFarraj O, AlZubi A, Tolba A (2018) Optimized feature selection algorithm based on fireflies with gravitational ant colony algorithm for big data predictive analytics. Neural Comput Appl. https://doi.org/10.1007/s00521-018-3612-0

9. Prabhakaran N, Sudhakar MS (2018) Fuzzy curvilinear path optimization using fuzzy regression analysis for mid vehicle collision detection and avoidance system analyzed on NGSIM I-80 dataset (real-road scenarios). Neural Comput Appl. https:// doi.org/10.1007/s00521-018-3553-7

10. Nagarajan SM, Gandhi UD (2018) Classifying streaming of Twitter data based on sentiment analysis using hybridization. Neural Comput Appl. https://doi.org/10.1007/s00521-018-3476-3

11. Rizk-Allah RM, Hassanien AE, Elhoseny M et al (2018) A new binary salp swarm algorithm: development and application for optimization tasks. Neural Comput Appl. https://doi.org/10.1007/ s00521-018-3613-z

12. Sun N, Lu Y (2018) A self-adaptive genetic algorithm with improved mutation mode based on measurement of population diversity. Neural Comput Appl. https://doi.org/10.1007/s00521018-3438-9

13. Pan X, Xue L, Li R (2018) A new and efficient firefly algorithm for numerical optimization problems. Neural Comput Appl. https://doi.org/10.1007/s00521-018-3449-6

14. Jing Y, Zhang Z (2018) A study on car flow organization in the loading end of heavy haul railway based on immune clonal selection algorithm. Neural Comput Appl. https://doi.org/10. 1007/s00521-018-3396-2

15. Jancy S, Jayakumar C (2018) Pivot variable location-based clustering algorithm for reducing dead nodes in wireless sensor networks. Neural Comput Appl. https://doi.org/10.1007/s00521018-3526-X

16. Gu L, Han Y, Wang C et al (2018) Module overlapping structure detection in PPI using an improved link similarity-based Markov clustering algorithm. Neural Comput Appl. https://doi.org/10. 1007/s00521-018-3508-Z

17. Ai Y, Li Z, Gan M et al (2018) A deep learning approach on short-term spatiotemporal distribution forecasting of dockless bike-sharing system. Neural Comput Appl. https://doi.org/10. 1007/s00521-018-3470-9

18. Arivudainambi D, Varun Kumar KA, Sibi Chakkaravarthy S (2018) LION IDS: a meta-heuristics approach to detect DDoS attacks against Software-Defined Networks. Neural Comput Appl. https://doi.org/10.1007/s00521-018-3383-7

19. Agasthian A, Pamula R, Kumaraswamidhas LA (2018) Fault classification and detection in wind turbine using Cuckoo-optimized support vector machine. Neural Comput Appl. https://doi. org/10.1007/s00521-018-3690-z

20. Sasikala C, Shoba Bindu C (2018) Certificateless remote data integrity checking using lattices in cloud storage. Neural Comput Appl. https://doi.org/10.1007/s00521-018-3546-6

21. Lokesh S, Malarvizhi Kumar P, Ramya Devi M et al (2018) An automatic Tamil speech recognition system by using bidirectional recurrent neural network with self-organizing map. Neural Comput Appl. https://doi.org/10.1007/s00521-018-3466-5

22. Dinesh Jackson Samuel R, Rajesh Kanna B (2018) Tuberculosis (TB) detection system using deep neural networks. Neural Comput Appl. https://doi.org/10.1007/s00521-018-3564-4

23. Al-Bashir AK, Al-Abed MA, Amari HK et al (2018) Computerbased Cobb angle measurement using deflection points in adolescence idiopathic scoliosis from radiographic images. Neural Comput Appl. https://doi.org/10.1007/s00521-018-3614-y

24. Ding N, Xu S, Song Z et al (2018) Using hardware counter-based performance model to diagnose scaling issues of HPC applications. Neural Comput Appl. https://doi.org/10.1007/s00521-0183496-z

25. Altameem T, Amoon M (2018) Crime activities prediction using hybridization of firefly optimization technique and fuzzy cognitive map neural networks. Neural Comput Appl. https://doi.org/ 10.1007/s00521-018-3561-7

26. Xing S, Ju J, Xing J (2018) Research on hot-rolling steel products quality control based on BP neural network inverse model. Neural Comput Appl. https://doi.org/10.1007/s00521-018-3547-5

27. Chandra Babu G, Shantharajah SP (2018) Optimal body mass index cutoff point for cardiovascular disease and high blood pressure. Neural Comput Appl. https://doi.org/10.1007/s00521018-3484-3

28. Abdel-Basset M, Gunasekaran M, Mohamed M et al (2018) A novel method for solving the fully neutrosophic linear programming problems. Neural Comput Appl. https://doi.org/10.1007/ s00521-018-3404-6 
29. Wang J, Xia L, Hu X et al (2018) Abnormal event detection with semi-supervised sparse topic model. Neural Comput Appl. https://doi.org/10.1007/s00521-018-3417-1

30. Wu Z, Zhang S (2018) Study on the spatial-temporal change characteristics and influence factors of fog and haze pollution based on GAM. Neural Comput Appl. https://doi.org/10.1007/ s00521-018-3532-z

31. Frank Vijay J (2018) Enrichment of accurate software effort estimation using fuzzy-based function point analysis in business data analytics. Neural Comput Appl. https://doi.org/10.1007/ s00521-018-3565-3

Publisher's Note Springer Nature remains neutral with regard to jurisdictional claims in published maps and institutional affiliations. 